\title{
Superficial and subcutaneous mycoses
}

\author{
Gianluigi Lombardi, ${ }^{1}$ Giuliana Lo Cascio, ${ }^{2}$ Stefano Andreoni, ${ }^{3}$ Elisabetta Blasi, ${ }^{4}$ Marco Conte, ${ }^{5}$ \\ Claudio Farina, ${ }^{6}$ Paolo Fazii, ${ }^{7}$ Silvana Sanna, ${ }^{8}$ Laura Trovato ${ }^{9}$ on behalf of AMCLI Study Committee for \\ Mycology (CoSM)
}

${ }^{1}$ Microbiology and Virology Laboratory, Grande Ospedale Metropolitano Niguarda, Milano; ${ }^{2}$ Microbiology and Virology Laboratory, Azienda Ospedaliera-Universitaria Integrata, Verona; ${ }^{3}$ Microbiology and Virology Laboratory, Azienda Ospedaliera-Universitaria Ospedale Maggiore della Carità, Novara; ${ }^{4}$ Department of Surgery, Medicine, Dentistry and Morphological Sciences; University of Modena and Reggio Emilia, Modena; ${ }^{5}$ Microbiology and Virology Laboratory; Grande Ospedale Metropolitano "Bianchi-Melacrino-Morelli", Reggio Calabria; "Microbiology and Virology Laboratory, Azienda Socio-Sanitaria Territoriale "Papa Giovanni XXIII", Bergamo; ${ }^{7}$ Clinical Microbiology and Virology Laboratory, Presidio Ospedaliero "Spirito Santo", Pescara; ${ }^{8}$ Microbiology and Virology Laboratory, Azienda Ospedaliera Universitaria, Sassari; ${ }^{9}$ Department of Biomedical and Biotechnological Sciences, University of Catania, Italy

\section{Classification of Mycoses}

Tegument mycoses are classified into three groups:

1) Superficial skin mycoses or superficial mycoses: infections sustained by fungi limited to the skin horny layer or the hair extrafollicular portion, without a significant inflammatory response in the host.

2) Skin mycoses (dermatophytosis) sensu stricto: fungal infections in which skin and its annexes, at the level of the keratinized layers, are involved with an evident immune response by the host

3) Mycosis of the subcutaneous tissue: fungal infections that mainly affect the subcutaneous tissue and secondarily, by contiguity, skin, bones and other tissues, with a strong immune response by the host.

Correspondence: Gianluigi Lombardi, Microbiology and Virology Laboratory, Grande Ospedale Metropolitano Niguarda, Milano, Italy.

E-mail: gianluigi.lombardi@ospedaleniguarda.it

Key words: Mycoses, subcutaneous, superficial, protocol.

Conflict of interest: The authors declare no potential conflict of interest.

Funding: None.

Availability of data and materials: All data are available within the text.

Received for publication: 3 June 2020 .

Accepted for publication: 5 June 2020.

${ }^{\circ}$ Copyright: the Author(s), 2020

Licensee PAGEPress, Italy

Microbiologia Medica 2020; 35:9156

doi:10.4081/mm.2020.9156

This article is distributed under the terms of the Creative Commons Attribution Noncommercial License (by-nc 4.0) which permits any noncommercial use, distribution, and reproduction in any medium, provided the original author(s) and source are credited.
1) Superficial skin mycoses or superficial mycoses

The clinical features of fungal superficial dermatites are:

1. Pityriasis versicolor due to Malassezia furfur.

2. Tinea nigra due to Exophiala werneckii.

3. White piedra due to Trichosporon species.

4. Black piedra due to Piedraia hortae.

\section{Clinical and epidemiological aspects}

Malassezia furfur is recognized as the main causative agent of Pityriasis versicolor, a chronic infection (colonization) of the stratum corneum, usually asymptomatic, distributed all over the world, mainly affecting young adults of both sexes. Skin colonization occurs following the increase in sebaceous secretion in pre-pubertal and pubertal age. In particular, about $70-80 \%$ of adults are normally colonized by $M$. furfur but, only in the presence of predisposing factors, fungi can switch from a saprophytic to a parasitic state. Excessive or altered production of sebum and excessive humidity of some body areas are among the factors contributing to infection, hyperproliferation and clinical manifestation.

High temperatures (summer season) and humidity favor the occurrence of pityriasis: in tropical areas, it has an incidence higher than 40-50\%, while in temperate climates it represents 5$10 \%$ of the dermatological pathologies. Pityriasis versicolor is characterized by finely flaking single lesions (pityriasis) which tend to converge ("map" aspect). They are usually asymptomatic and itchy only in a small percentage. The infection course is generally chronic, with frequent relapses. The lesions are generally located in the upper part of the trunk, neck and upper limbs. The infection can extend to the abdomen, groin and thighs. Lower limbs and face are rarely affected. The same patient may have differently shaped lesions, while the color depends on the scales thickness, infection severity, level of sun exposure. Before sun exposure they have a "chamois" color; sun exposure cannot pigment these lesions due to azelaic acid, a fungal metabolite, which enzymatically blocks the melanin synthesis process (hence the name "versicolor"). In fair-skinned subjects, the lesions may appear darker than normal, while on 
black people they may present as depigmented areas (Pityriasis versicolor alba).

Fungi belonging to the Malassezia genus can cause, in addition to superficial infections, systemic infections especially in immunocompromised patients. In particular, systemic infections may affect preterm infants hospitalized for a long period in the Neonatal Intensive Care Unit (NICU), who receive parenteral lipid hyperalimentation. In fact, despite the evidence of the high degree of skin colonization in young adults compared to prepuberal children, some studies have reported a high degree of colonization in the NICUs. These conditions urge the need to implement preventive measures in order to reduce the transmission of yeast and to prevent the possibility of neonatal sepsis by Malassezia in immunologically immature children.

Tinea Nigra is a superficial mycosis of the horny layer on the hand palm-plantar regions, feet soles and other parts of the body, presenting with painless, hyperpigmented macules (from dark brown to black); hyperpigmentation is due to the accumulation of melanin-like substances. The infection is caused by Exophiala werneckii (Hortaea werneckii or Phaeoannellomyces werneckii), a fungus present in tropical regions. Infection sources are generally soil, compost, and rotting wood. Tinea nigra is more frequent in children and adolescents; however, all ages can be affected.

Trichosporon is recognized as the causative agent of White piedra, infection of the hair, beard hair, pubis and armpits. The causative agent, previously identified as T. beigelii, is now considered T. asahii, along with other five species: T. ovoides, T. inkin, $T$. mucoides, T. asteroides and T. cutaneum. If all the species can be considered as possible agents of White piedra, T. asahii is regarded as the most closely related to this pathology. Detected in temperate areas of Europe, Asia, North and South America, this pathology is more common in young adults. Special cosmetics or lotions can act as vehicles. T. inkin can also give rise to localizations at pubis hair site, with diabetic glycosuria being a predisposing factor.

Black piedra is an asymptomatic superficial hair infection caused by Piedraia hortae, an ascomycete capable of forming hard, black nodules adhering to the hair, beard hair and pubic hair. This pathology is common in tropical regions in relation to higher temperatures and humidity (South and Central America and Southeast Asia). It generally affects young adults and can be epidemic in the same family (sharing of brushes and combs).

White piedra is characterized by the formation of whitish nodules $(1-1.5 \mathrm{~mm})$ on the hair shaft, mainly in the distal part; the nodules are particularly adherent and, in some cases, can form sheaths around the hair extremity. It may happen that the hair breaks near the follicular exit. Shaving generally solves the infection. The surrounding skin is not affected.

Tinea Nigra is characterized by macular lesions, with discrete, round or irregular edges, which appear as areas of skin hyperpigmentation varying in color from brown to black. The typical lesion is generally single, although multiple forms may be present. Palmary and plantar surfaces are involved, with possible extensions to the fingers. Areas such as neck or chest are rarely affected. Dimensions vary from a few millimeters to a few centimeters in diameter. Patients are generally asymptomatic, although cases with itchy symptoms were reported. A differential diagnosis with Addison's disease, dysplastic nevus, malignant melanoma, and syphilis may be necessary.

\section{Diagnostic aspects}

Laboratory diagnosis is mainly based on direct microscopic examination, isolation in culture and identification.

\section{Direct microscopic examination}

The direct microscopic examination represents a rapid and specific test for the recognition of fungal elements in clinical samples. Many of the materials under consideration should be initially clarified (see dermatophytoses). In case of suspected Pytiriasis versicolor, the direct microscopic examination (scotch test) with lactophenol cotton blue (mount) is essential for the diagnosis and highlights double-contoured yeast cells, cluster-like arranged and squat hyphae with a "spaghetti and meatballs" like appearance. In case of Black piedra suspicion, hair should be examined using potassium hydroxide $10 \%$ mount or Calcofluor White staining. The nodules are made up of a pigmented mass with a central stromal area containing asci.

\section{Mycological cultures}

M. furfur isolation requires the use of particular media, being a lipophilic yeast that needs mineral oils or long chain fatty acids for growing. The most practical cultivation method consists in using Sabouraud Dextrose Agar added with 1\% cycloheximide covered, on the surface, by a thin film of olive oil. In this case the growth is rapid (4-5 days) at $35-37^{\circ} \mathrm{C}$, but very difficult if the incubation takes place at $25^{\circ} \mathrm{C}$. Another useful medium for the isolation of Malassezia is m-Dixon agar which contains Tween 40 and glycerol monooleate. In addition, chromogenic media currently on the market, besides isolation, allow to more easily highlight the presence of different Malassezia species in the same clinical sample, thus starting a presumptive identification. However, the diagnosis, strongly suspected on a clinical basis, is usually confirmed by the positivity of the direct microscopic examination.

In case of suspected Black piedra, the hair fragments should be inoculated on primary media, such as Sabouraud's Dextrose agar. $P$. hortae colonies after two to three weeks of incubation are dark, brown black.

\section{Identification}

The identification of the different species belonging to the genus Malassezia is conventionally performed studying macroand microscopic characteristics (different morphology and cell sizes) and the different ability to use lipid sources such as Tween 20, 40, 60 and 80 (Tween test). These methods are often difficult to use in laboratory routine, due both to their complexity (Tween test) and to the morphological similarity of the different species. In this regard, several molecular methods have been developed, such as DNA sequencing (LSU rRNA and 26S rRNA gene) and PCRRFLP (restriction fragment length polymorphism), which can provide a reliable identification at the species level. These techniques are usually used for the identification of strains isolated from patients with deep infections.

In the last decade, MALDI-TOF (Matrix Assisted Laser Desorption Ionization Time-of-Flight) technology has been developed and used for the rapid identification of bacteria, yeasts and filamentous fungi. The technique consists in mixing the sample pretreated with a matrix. The mixture applied on a plate is irradiated by a pulsed laser beam. The matrix absorbs the laser light and vaporizes together with the sample acquiring an electric charge (ionization). The ionized microbial molecules are accelerated, on 
the basis of mass/charge ratio, in a TOF mass analyzer ("time-offlight" = flight time) and its quantity is measured. The protein profile obtained is characteristic for the fungal species under examination. Currently VITEK MS (bioMérieux) and MALDI Biotyper CA System (Bruker Daltonics) are the two systems marketed and approved by the American Food and Drug Administration (FDA) for the identification of bacteria and yeasts in culture and, in the case of the first system, for filamentous fungi, too.

\section{2) Cutaneous mycoses sensu stricto (dermatophytoses)}

Dermatophytes are fungi capable of feeding on keratin (keratinophilic) which, as known, represents the main component of human skin, hair and nails. As even animals'skin, fur, horns and hooves, as well as birds' feathers and plumes, are made of keratin, animals can also be affected by dermatophytoses. Animal dermatomycoses, and in particular dermatophytoses, are considered zoonoses, a term indicating all diseases that can be transmitted, directly or indirectly, from vertebrate animals to humans and viceversa. The infection caused by Dermatophytes is called dermatophytosis or dermatophytia and can therefore affect both humans and animals, essentially mammals and birds. By definition, only those species that are pathogenic to animals and humans can be considered dermatophytes. The dermatophyte group includes three anamorphic genera: Epidermophyton, Microsporum and Trichophyton. For some species the "perfect forms", or teleomorphic, are also known, included in the genus Arthroderma. Dermatophytoses are internationally called with the Latin term tinea (= worm or insect larva), followed by the specification, also in Latin, of the parasitic anatomical site (tinea unguium, tinea corporis, tinea capitis, etc.). Dermatophytoses are superficial mycoses, unable to parasitize the dermis and spread to the internal organs. However, under particular immunological conditions, deep forms of dermatophytoses may be noted. Dermatophytoses are among the most frequent mycoses in human pathology; some forms, such as tinea pedis, seem to affect very high percentages (with a prevalence ranging from 10 to $70 \%$ ) of subjects residing in the industrialized areas of the world. Pets, especially dogs and cats (but also rabbits and guinea pigs) represent the main reservoirs of infection, also because they are often "healthy" carriers of conidia that can be transmitted to humans by both direct and indirect contact. In other cases, the reservoir is represented by farm animals (cattle, pigs, horses), while infections in wild animals have also been reported. Furthermore, fungal dermatitis with skin localization can also be the cutaneous expression of disseminated mycoses due to, for example, Candida spp., Cryptococcus spp. and Fusarium spp. Dermatophytoses are very common fungal infections affecting both humans and animals. The epidemiology of dermatophytoses can be correlated to various factors, some intrinsic to the pathogen (zoophilia and anthropophilia, strain virulence), others linked to the host age and sex, others extrinsic such as, for example, climatic factors.

\section{Infection reservoirs}

Infected humans or animals can be reservoirs of infection. In dermatophytoses caused by geophilic species, soil represents the infection source.

\section{Seasonality}

Hot and humid climate seems to favor the onset of dermatophytoses, especially of some clinical forms (tinea pedis, tinea cruris). For this reason, especially in Southern European countries, the highest number of cases is reported during the summer.

\section{Routes of infection}

Infection can occur both directly (as for tinea cruris, which can also be classified as a sexually transmitted disease) and indirectly (through hats, clothes, sheets, combs, razors, backs of cinema seats, etc.) as well as for autoinoculation (as for tinea unguium). The structures associated with the infection are the arthroconides and chlamydospores, found inside or outside hair or skin scales. Those of certain species can persist in the environment for several months, maybe years, especially if present inside hair or scales.

\section{Geographical distribution of cases}

Zoophilic dermatophyte infections are more frequent in rural environment and can be related to working activity (shepherds and breeders). Farm animals that most frequently host dermatophytes on their integument are cattle (usually associated species: T. verrucosum), rabbits (mainly associated species: T. mentagrophytes) and pigs (mainly associated species: M. nanum). Conversely, a species of zoophilic dermatophyte, $M$. canis, is frequently associated with pets like dogs, rabbits (such as dwarf rabbits, which have now become pets) and especially cats, although apparently asymptomatic. For this reason, M. canis is often the cause of dermatophytoses even in children living in urban centers. Anthropophilic dermatophyte infections can have specific geographic patterns, such as tinea imbricata caused by T. concentricum that is observed only in some areas of the Pacific Ocean and Central-South America. Conversely, some species like $T$. rubrum, T. tonsurans and E. floccosum are known to be ubiquitous. Anthropophilic species can cause recurrent epidemics in the community or family environment such as those caused by $T$. tonsurans and T. violaceum, as well as by other species, such as T. rubrum. Anthropophilic fungi can then persist in particular environments like swimming pools and gyms, where they usually cause tinea pedis and tinea unguium, or in showers and bathrooms, and more generally in rooms shared by a large number of subjects such as barracks, ships factories, kindergartens and schools. In Italy and Southern Europe, dermatophytic infections are more frequently caused by (in order of prevalence): $T$. rubrum, M. canis, T. mentagrophytes and E. floccosum. As already mentioned by some international authors, we recently observed dermatophytoses cases due to species disappeared in our country: this is due to the arrival of non-European people, and the isolated species are typical of the areas of origin of these subjects (i.e. T. violaceum, M. audouinii or T. soudanense). Generally speaking, in developing countries, due to both the precarious hygienic conditions of life and promiscuity, cases due to anthropophilic fungi are more often detected, whereas in industrialized countries, with some exceptions, we can observe forms due to zoophilic dermatophytes. 


\section{Age}

Tinea capitis is a form of dermatophytosis that is nearly always described in children. In fact, in the sebum of prepubescent subjects medium-chain fatty acids with a proven antifungal activity are not yet present. Conversely, among adults, tinea cruris, tinea pedis and tinea unguium are more frequently observed. Tinea corporis is described in all ages of life, including the neonatal one.

\section{Sex}

Some dermatophytoses are more frequently associated with male sex (tinea cruris, tinea barbae), while tinea capitis is associated with the adult female sex. They occur in particular in the postmenopausal period, due to the reduction of a significant part of saturated fatty acids inhibiting the development of dermatophytes, caused by the hormonal modifications typical of this stage of life.

\section{Profession}

Sometimes dermatophytoses caused by zoophilic Dermatophytes are detected in people involved in cattle care. Even veterinarians, for obvious reasons, are exposed to the risk of infection. Miners also frequently present dermatophytoses due to anthropophilic Dermatophytes, whose development is mainly sustained by mines microclimate. Athletes can also be affected by these infections. This might be due both to the use of footwear and type of activity (involving high sweating, changes in skin $\mathrm{pH}$, microtraumas) that would favor the development of Dermatophytes. Additionally, those microclimates (hot-humid climate present in swimming pools and gyms) are ideal for Dermatophytes spread. In particular, it could also be due to sports practices that involve prolonged contact between athletes (the socalled tinea gladiatorum, observable among wrestlers).

\section{Pathogenetic and clinical aspects}

The infection caused by Dermatophytes is usually limited to the stratum corneum of the epidermis, nails, and hair. The colonization and invasion process of the stratum corneum begins with arthroconidia adherence to the corneocytes. Adherence is followed by the germination, the subsequent penetration of the germ tubes into the stratum corneum and finally the formation of hyphae, starting from the arthroconidia, with a rapid centrifugal diffusion in every direction. Keratin seems then to be degraded by Dermatophytes by breaking the disulfide bridges by the means of sulphites secreted in the substrate. Keratin is then further used by dermatophytic extracellular proteases (keratinases, elastases, collagenases) and other enzymes (lipases, ureases), whose production varies in relation to the parasitic species (maximum for the zoophilic dermatophytes, such as T. mentagrophytes) and skin microenvironment conditions $\left(\mathrm{CO}_{2}\right.$ concentration). Fungi implant on the skin and their subsequent colonization also seem to be favored by their ability to release some substances with antibiotic properties, such as streptomycin, fusidic acid and some beta-lactams. Various factors may favor the development of Dermatophytes on the skin, partly influencing their implant on the stratum corneum, as well as the subsequent spread. The anatomical-physiological aspects, the climate, social, iatrogenic, and pathological aspects are described among the favoring factors. On the other hand, the organism resists the infection through non-specific and specific defense systems.

Dermatophyte related infections are called dermatophytoses. The various clinical forms are internationally indicated with the Latin term tinea followed by the specification of the parasitic site. The manifestations are related to both the involved species and the site of infection, as well as the host's immune status, and numerous other factors (age, sex, iatrogenic factors, etc.). In human pathology, the most often applied clinical classification of dermatophytoses is the one that assesses the eventual parasitic presence on skin appendages. According to this classification, there are two large groups of dermatophytoses: dermatophytosis of the glabrous skin (tinea corporis) and dermatophytosis of the skin appendages (hair, nails). Other clinical manifestations, such as deep dermatophyte infections, dermatophyte disease, Majocchi's granuloma tricophyticum and other forms of granuloma tricophyticum, as well as dermatophyte mycetomas, are rarely observed.

\section{Diagnostic approach: the mycological examination}

The clinical diagnosis of dermatophytoses in full-blown cases does not present particular difficulties. Sometimes, the clinical presentation is not typical or its aspect can be modified by the use of incongruous therapies. In these cases, a laboratory investigation, that does not involve excessive technical difficulties, is mandatory and diriment. However, it is better to request a consult by the Microbiology Laboratory in all cases of suspected dermatophytosis, as only a duly performed mycological examination can confirm the diagnostic suspicion and provide significant information from an epidemiological point of view. The laboratory diagnostics of dermatophytoses is based on both direct mycological examination and mycological culture, as well as on some other additional tests.

\section{Samples collection}

The mycological tests can be adequately prepared only if the clinical sample is properly collected, being collection one of the most critical stages. The patient should also not have applied any local antifungal therapy. Sample collection must be preceded by a careful cleansing and performed possibly a few days after the last medicamentous treatment. Before sampling, skin or annexes have to be wiped with $70 \%$ ethyl alcohol (to reduce also the local bacterial load).

Sampling must be carried out in the lesions areas where live hyphae can be found, namely:

A) peripheral border of the herpes circinatus

B) vesicles top

C) scales of interdigital spaces

D) broken hair and scales in the alopecic area

E) kerion pus

F) fragments of infected nails, taken in the area of contact with the healthy part.

Collection can be performed by a sterile scalpel; scales should be collected in a sterile Petri dish or on microscope slides. Sometimes, a sterile gauze or a piece of self-adhesive plastic velvet (UV sterilized) can be used. The rough surface, used for the collection, is then placed on a medium in Petri dishes. For microscopic examination only, it is suggested to use a simple transparent adhesive tape to be applied, after collection, on microscope slides. 
Sterile forceps are used to collect broken hair, paying particular attention to those apparently parasitized (fragile, dull).

Collection of nail fragments can be problematic as fungi are usually located between a portion of a sick and healthy nail, in a site not easily accessible. For the collection, a cutter is generally used to remove the entire portion of the detached lamina that cannot be utilized for the mycological examination, as it is usually heavily contaminated with saprophytic filamentous fungi. When using a sharp curette, the nails in contact with the healthy portion have to be scratched. Alternatively, a small hole can be made with a bur on the proximal portion of the lamina overlying the onychosis area. If wet exudative lesions are present, a sterile swab might be used. In case of suspected Pityriasis, a transparent adhesive tape applied to the skin can be used to collect the scales and, subsequently, placed on a microscope slide pretreated with a clarifying liquid.

\section{Storage and transportation of clinical specimens}

Scales and skin appendages can be stored for many days, without danger of deterioration, before being forwarded to the laboratory for mycological tests. In these cases, storage must be carried out in sterile containers (for example a test tube, an empty Petri dish; use of two wrapped slides must be discouraged) avoiding the hermetic sealing of the containers and the addition of liquids, as an excessive humidity could encourage the development of bacteria and contaminating fungi. Conversely, the samples of wet biological material (pus, etc.), should be processed within few hours of collection.

\section{Direct microscopic examination}

Direct mycological examination is the first step if a suspicion of Dermatophyte or any other fungus in the clinical samples arises. On the other hand, microscopic examination allows only a presumptive diagnosis of dermatophytosis and presents a variable sensitivity in relation to the observer's skills and the infecting species. The fungal hyphae investigation may be negative in the event of an incorrectly performed examination and, on the contrary, it can happen to have a positive direct mycological examination with a negative culture in case nail containing non-viable hyphae are analyzed, therefore visible only on a direct examination. In order to make improve the visibility of fungal elements, the specimen clarification (scales, hair, nails) is performed by placing a drop of clarifying solution on a microscope slide and dipping the small fragments of keratin material, subsequently covered with a coverslip. There are several clarifying substances that can be used; however, it is usually preferable to use potassium hydroxide 10$40 \%$ in aqueous solution or in water and $20 \%$ glycerol. A $40 \%$ solution is recommended for the clarification of hard and compact material, such as nails. Another liquid that can be used is sodium hydroxide $10-20 \%$ with or without the addition of $5 \%$ glycerol, or $10 \%$ sodium sulphide. Dyes (blue-Parker, cotton-blue, toluidine blue) can be added to the clarifying liquids, thus allowing to better highlight the fungal structures. The clarifying substance should be allowed to act from 30 minutes to a few hours. For the sample dissociation and lipids emulsion, it can be useful to heat the clarified sample over the flame of a Bunsen burner for a few seconds or leave it in an incubator at $51-54^{\circ} \mathrm{C}$ for 1 hour. The preparation should be observed under an optical microscope at 100X and at
400X. Dermatophytes are recognized by the presence of septate, hyaline hyphae and arthroconidia of various sizes. The presence of artifacts or extrafungal structures (pseudofungal mosaic, lipid drops, crystals, tissue fibers, etc.) can complicate the examination, which requires expertise, attention and time. Another possibility is the use of clarifying substances with the addition of fluorochromes, such as Calcofluor white in potassium hydroxide $10 \%$ or acridine orange $(1: 10.000)$ or the technique with Congo red in potassium hydroxide $20 \%$. In these cases, the observation must be performed with the fluorescence microscope. Depending on the infectious dermatophytic species, three basic types (and some variants) of hair parasitism are here below described.

\section{Ectothrix type}

The genus Microsporum fungi, once penetrated into the bulb, again pierce the lamellae of the hair cuticle proliferating around it. Therefore, chains or spore clusters are observed outside the hair shaft.

\section{Endothrix type}

The mycelial filaments penetrated into the hair are divided into 5-8 $\mu$ diameter spore chains (large spore endothrix parasitism) and completely fill it ("nuts sack" appearance). The shaft is more severely damaged compared to "ectothrix type" parasitism and breaks right at the ostium exit.

\section{Favic type}

The fungus ( $T$. schoenleinii) invades the infundibulum of the pilo-sebaceous unit, produces a large number of hyphae that, mixed with keratinocytes, thicken around the hair becoming macroscopically visible: these are the scutules typical of this types of ringworm. When later the fungus enters the hair in its proximal part, it produces hyphae without spores, while empty hyphal residues containing air can be detected in the distal part. Hair appears less damaged, compared to parasitized hair in the manner described above and can grow normally without breaking. The chronic progress of the lesions can often result in scarring alopecia.

\section{Mycological culture}

Cultures should always be performed in parallel with the direct mycological examination. Cultivation of clinical material can confirm the result of the direct examination and, by identifying the parasitic species, provide useful epidemiological data. To properly perform a cultural examination, it is mandatory to use suitable culture media guaranteeing the optimal fungi growth, thus limiting as much as possible the chance of contamination by microbial agents (bacteria and filamentous fungi) which, developing more rapidly, could mask or inhibit their development. Media in Petri dish, in glass or plastic tubes can be used, either directly prepared in laboratory from standard formulas, or purchased from well-known producers. Suitable media are the following. 


\section{Sabouraud Dextrose Agar (SDA)}

Most mycologists believe that, for Dermatophyte culture, it would always be convenient to use this medium, together with, if necessary, a more selective medium. In fact, the macro-morphology of the dermatophytes cultures undergo substantial changes in relation to the medium used for the growth. Therefore, for this purpose, it is preferable to use a universally known and used medium, such as SDA.

\section{SDA added with chloramphenicol and gentamicin}

This medium can limit bacterial growth, not preventing filamentous fungi growth.

\section{SDA added with chloramphenicol and cycloheximide (actidione)}

This is a selective medium for dermatophytes: bacteria are inhibited by antibiotics, while cycloheximide prevents the growth of contaminating filamentous fungi.

\section{Dermatophyte Test Medium (DTM) (Taplin 1969)}

This medium contains chlortetracycline, gentamicin, cycloheximide and phenol red as $\mathrm{pH}$ indicator. Proteolysis caused by dermatophytes growth determines the production of ammonium ions which raise $\mathrm{pH}$, with a consequent medium color change from yellow to red due to phenol red activation.

\section{Dermatophyte Identification Medium (DIM)}

Proposed by Salkin et al. in 1997 for the presumptive identification of Dermatophytes, it contains dextrose, neopeptone, cycloheximide, penicillin, streptomycin and bromocresolpurple as $\mathrm{pH}$ indicator. In the presence of Dermatophytes, the medium color changes from green-blue to intense purple. The skin scales, nails or hair must be placed on 4 or 5 points on of the medium, possibly in contact with glass or plastic as here fungi develop better. It is a good practice to use contemporaneously a selective medium for dermatophytes (e.g. SDA with the addition of antibiotics and cycloheximide) and a medium where also yeasts and molds, which can be involved in infectious processes, can develop.

\section{Incubation}

Except where there is a suspicion of an infection caused by $T$. verrucosum (zoophilic species responsible for the greatest number of cases of bovine dermatophytosis), which grows well at $37^{\circ} \mathrm{C}$, in all other cases incubation takes place at $27^{\circ} \mathrm{C}$ or at room temperature for at least 4 weeks. Almost all Dermatophytes develop within this lapse of time, although some fungi, such as T. ochraceum, require up to 2-3 months of incubation. Culture examination is carried out starting from the second day of incubation and should possibly be performed two or three times a week. The culture results will be reported as negative only if at least four weeks have passed since inoculation. Small portions of aerial mycelium of the dermatophytic colonies must be taken and placed on new plates or new tubes if pollution by contaminating fungi or bacteria during the incubation period is detected.

\section{Identification}

Identification is based on macro-micromorphological examination of the colonies. Many times the most common Dermatophytes are identified directly from the primary media.
Sometimes, however additional tests that may meet the microbiologists' requirements, must be performed.

\section{Macroscopic examination}

Starting from the first day of colony development, the following macroscopic features should be observed:

- colony morphology (cottony, fluffy, velvety, floury, grainy, chalky, wrinkled, mammoth, crater-like, cerebriform)

- colony topography

- colony color (recto and verso)

- pigment production, that is verified by observing the plate bottom (verso).

\section{Microscopic examination}

The examination must be performed in the following way:

- colony fragment collection

- scotch technique

- slide culture

In the first instance, Dermatophytic species identification is carried out with the microscopic observation of the reproductive organs (macroconidia, microconidia and chlamydospores) and/or of the ornamental organs (pectinate hyphae, spiral hyphae, nodular organs, candlesticks, racquet hyphae).

More in detail, the following features must be searched in the microscopic preparation.

\section{Epidermophyton spp.}

Microconidia are always absent; macroconidia are clavate and smooth, 20-60 $\mu$ long, 4-13 $\mu$ wide; the cell wall is thin or moderately thick with 1-9 septa. Chlamydospores may be present; of the two species E. stockdalae and E. floccosum, only the latter is pathogenic to humans.

\section{Microsporum spp.}

Microconidia, usually scarce or absent, are sessile or pedunculated and clavate, single or gathered in branches as in M. racemosum. Macroconidia have thick, rough walls that can be asperulate, echinulate or warty; they can be fusiform or ovoid (as in $M$. nanum) or cylindrical (as in M. vanbreuseghemii); septa can be from 1 to 15 . Macroconidia dimensions are 6-160 $\mu$ (in length) 6$25 \mu$ (in width). Chlamydospores, pectinate and racquet hyphae (in some species) may be present.

\section{Trichophyton spp.}

Microconidia are numerous, globose, piriform or clavate, sessile or pedunculated, single or gathered in clusters. Macroconidia, when present, are smooth, usually thin-walled, with a number of septa varying from 1 to 12 , single or in clusters and can be elongated in the shape of a pencil, clavate, fusiform or cylindrical. Macroconidia length ranges from 8 to $86 \mu$, width from 4 to $14 \mu$. Chlamydospores may be present. In some species, there may be ornamental organs, such as spiral hyphae, candlestick hyphae, racquet hyphae, nodular organs.

\section{Identification}

\section{Additional tests}

If the previous examinations have not highlighted the struc- 
tures necessary for the identification to the species level, specific tests should be performed by reference laboratories. In particular, when a Dermatophyte of the genus Trichophyton is suspected, formulated in a different media way can be used (Trichophyton Agar) according to the nutritional requirements, in terms of supply of vitamins and amino acids, essential or partially necessary for the different species development :

- Casein Agar Base (negative control without vitamins)

- Casein Agar Base + inositol (need for inositol)

- Casein Agar Base + inositol + thiamine (for the growth of $T$. Verrucosum)

- Casein Agar Base + thiamine (need for thiamine for the growth of $T$. tonsurans and T. Violaceum)

- Casein Agar Base + nicotinic acid (need for nicotinic acid for the growth of T. equinum)

- Ammonium nitrate Agar Base (negative control)

- Ammonium nitrate Agar Base + histidine (need for histidine for the growth of T. megninii).

It is then possible to carry out other tests, among which we recommend:

- Urease test. This method allows to test the production of urease by some species of Dermatophytes. The fungal isolate is inoculated on Christiansen's Urea Agar and observed daily in order to detect any change in color from yellow to red, caused by an alkaline reaction, produced by some microorganisms. This test is used for the identification of some species of Dermatophytes belonging to the genus Trichophyton: T. mentagrophytes and T. tonsurans become positive in 7 days, while T. rubrum is urease negative.

- Test with "BCP-milk solids-glucose-agar"

- In vitro hair perforation test

- Pigment test in Dextrose Potato Agar

- Test with rice grains medium

- Test with BCP-casein-yeast extract-agar medium

- Test for macroconidia development

- Study of the perfect or sexual form.

\section{Mass spectrometry (MALDI-TOF)}

Even for Dermatophytes, technology based on mass spectrometry has been developed and used for the rapid identification of isolates. Analysis of published studies regarding the use of this technology shows that there are some critical factors that can influence this process, such as the relatively slow growth, production of pigments, culture medium, extraction procedure, tool used and the library of spectra that should certainly be implemented for these fungi. Actually, those that seem to have the greatest impact on the identification accuracy are the extraction phase (best results with complete extraction using formic acid-acetonitrile) and the possibility of using "home-made" databases implemented with spectra obtained from clinical isolates and reference strains. In the presence of these conditions, identification of dermatophytes by MALDI-TOF technology is certainly more economical, rapid and sometimes offers an accuracy comparable to that of DNA sequencing.

\section{Molecular tests}

Over the last few decades, molecular techniques have been developed allowing for the identification of Dermatophyte species both from culture and directly from clinical material. A multiplex
PCR method seems particularly promising as it allows for the detection and identification of dermatophytes directly from skin, hair and nails. Actually, it could be useful to support the diagnosis of onychomycosis in those patients who have started antifungal treatments, without mycological assessment and clinical improvement, for whom conventional diagnostics could be problematic. In these specific cases, the use of this method would certainly allow for a more rapid and sensitive diagnosis, thus avoiding further sampling after about two to three months from the therapy suspension and favoring a more appropriate use of the antifungal drugs.

\section{Other diagnostic tools: Wood's light}

Wood's lamp emits long-wave (UV-A) ultraviolet light (365 $\mathrm{nm}$ ). Some Dermatophytes emit bright (M. canis, M. audouinii, $M$. ferrugineum) or pale green (T. schoenleinii) fluorescence in vivo and not in vitro, which can be seen in dark environments. It is useful in the control of epidemics and subclinical infections, especially in cases of microsporic and favic tinea capitis and in the control of the individual response to treatment.

\section{Immunological methods}

Currently, the only suitable immunologic test is the intradermoreaction with trichophytin, a water-soluble extract derived from the culture of different Dermatophytes; the procedure is similar to that used for the tuberculin test. This test is being seldom used, due to the limited help it provides in interpreting the clinical picture. It can be useful only for epidemiological purposes.

\section{Histological examination}

Rarely used for diagnostic purposes, it can be useful in case of granulomatous or deep lesions. In case of tinea corporis, fungal hyphae localized on the stratum corneum can be detected (with PAS staining or other techniques, such as silver staining); sometimes, especially in infections caused by zoophilic fungi, papules and vesicles with significant dermal infiltrate can be observed. In chronic infections caused by anthropophilic Dermatophytes, a marked hyperkeratosis can be noticed; a parakeratosis (presence of nuclear structures in the corneocytes, which are not totally keratinized) can often be observed. Therefore, Parakeratosis can be interpreted as a defense mechanism activated against Dermatophytes. In fact, parakeratosis is a sign of increased cell turnover, a mechanism by which the host attempts to eliminate the infectious agents. In kerion, lymphocytes, plasma cells, eosinophils and neutrophils dermal infiltrates are observed. Sometimes penetration of hyphae into the dermis and signs of perifollicular and perivascular inflammation are noted. In T. rubrum folliculitis, fungal elements in the follicles and signs of a foreign body reaction are detected, with giant cells in the dermal infiltrate; sometimes, real granulomas can develop. In deep clinical forms, including Majocchi's trichophitycum granuloma, inflammatory and granulomatous features dominate the histological picture. According to some authors, in course of onychomycosis, the histological examination of the ungueal lamina is more sensitive than conventional mycological investigations for the diagnosis. In addition, the histological examination of the infected nails would be 
useful especially in the presence of mixed infections caused by dermatophytes and molds or yeasts.

\section{3) Mycosis of the subcutaneous tissue}

As for the pathogenesis, subcutaneous tissue mycoses are due to the direct and traumatic penetration into the subcutaneous tissue of fungal conidia of telluric mycetes, mostly through minor or (not apparent) invisible traumas. This explains why the subjects most often affected by such pathologies are those living in rural areas, mainly in the warm regions of the world (tropical and subtropical areas) where they carry out work or recreational activities in contact with the ground. However, any subject who, for any reason, both recreational and professional, is repeatedly in contact with soil and plants, can be affected in any part of the World.

\section{Classification}

The following is the description of well-defined clinical pictures of subcutaneous mycoses (of exclusive tropical relevance) mainly observed in the tropical areas, (and therefore considered imported mycoses in our country), such as:

A. Mycetomas

B. Chromoblastomycosis

C. Zygomycosis due to Entomophtorales

D. Lobomycosis.

Other pathologies, although rarely, can be detected in our country:

E. Sporotrichosis

F. Phaeohyphomycosis

G. Hyalohyphomycosis

H. Zygomycosis due to Mucorales

I. Subcutaneous dermatophytosis.

Finally, it is possible to observe subcutaneous localizations such as secondary manifestations of mycoses due to yeast, filamentous mycetes (hyaline or dematiaceous) and dimorphic fungi.

\section{Epidemiology and clinical features}

\section{A. Mycetoma}

Mycetoma is an infection characterized by the presence of a very slowly evolving granuloma, due to fungal penetration into the tissue, usually mediated by pointed and penetrating objects. In some cases, but not all, the formation of sinus tracts with pus discharge, inside which the "grains", fungal agglomerates mixed with the guest organic substances, can be present. Morphological and color evaluation of these grains, besides their texture, allows for a presumptive etiological diagnosis of mycetoma. The first description of mycetomas dates back to the Sanskrit religious work "Atharva Veda" (2000-1000 BC) where an illness called 'padavalmika' (anthill foot) is mentioned. The first "modern" description is attributed to Gill who, in 1842, reported the observation in the district of Madurai of some cases located in the lower limbs (hence the name "Madura foot" and "Maduromycosis"), although the most detailed presentation is attributed a few years later (1844-45) to Godfrey Garrison, surgeon in Madras who described four cases. Carter is the physician who in 1860 described the infectious character of mycetoma. He, with his "degenerative theory" of "black grains" from "white ones" described in the monograph titled "Mycetoma or the fungus disease of India", detailed all clinical and etiological aspects with a very precise description of Madurella mycetomatis. There are many synonyms for mycetoma: Madura foot, Maduromycosis, Godfrey and Eire disease, Morbus tuberculosis pedis, Morbus pedis endophyticus, Endemic degeneration of bones and foot, Perforating foot ulcer. However, the only recognized definitions are Mycetoma and Madura foot.

The limbs acral parts are usually infected, although sometimes other body sites have been involved. In fact, besides skin and subcutaneous tissue, muscles and bones may also be affected. The mycetoma etiological agents are $M$. mycetomatis (especially in Africa and India: $70 \%$ of the cases with "black grains"), M. grisea ("black grains") and Scedosporium aurantiacum (especially in the USA, with "white grains"). Less frequently, other dematiaceous fungi are recognized, such as Pyrenochaeta romeroi, Phialophora verrucosa (South America), and Leptosphaeria senegalensis (Africa); even more anecdotally Curvularia spp., Exophiala jeanselmei, Cylindrocarpon cyanescens, C. destructans, Neoscytalidium dimidiatum, Neotestudina rosatii, Corynespora spp and Polycytella spp. are reported as a cause of infection. Less frequently, mycetoma is caused by hyaline fungi, such as Acremonium falciforme, A. kiliense, A. recifei, Aspergillus nidulans, Fusarium solani (these species cause the white grains).

Mycotic mycetomas have been reported almost everywhere, but the highest incidence has been detected in the tropical and subtropical regions of the world, in particular in the Indian Subcontinent, Africa and South America. India and Sudan are the countries with the highest incidence. In Africa, some cases were also reported in Egypt, Senegal, Mauritania, Kenya, Niger, Nigeria, Chad, Cameroon, Madagascar and in the countries of the Horn of Africa (Ethiopia, Eritrea, Djibouti, Somalia). In Latin America, some cases have been reported mainly in Mexico, Venezuela, Colombia, but also in Argentina and Brazil. Cases in North America (USA) are sporadic. Eumycetomas have been found also in: Turkey, Lebanon, Saudi Arabia, Iran, Philippines, Japan, Sri Lanka, Thailand. In Europe, eumycetomas have been detected in Germany,

The Netherlands and in the Balkan Countries, mainly. Those affected live in rural areas and carry out particular work activities (farmers, woodsmen, gardeners, etc.), with the male sex being the most affected.

\section{B. Chromoblastomycosis}

It is a chronic, ubiquitous mycotic infection of the subcutaneous tissues and skin caused exclusively by dematiaceous fungi, that live as saprophytes in the soil, or are located on the organic material; they develop following a local trauma, usually of minor relevance. From a mycological and histological point of view they are characterized by the presence of the so-called "sclerotia or Medlar bodies". In 1913, Pedroso identified from a lesion, later called chromoblastomycosis, a black fungus,until then unknown, that was named Fonsecaea pedrosoi after him. The first description (1915) of the sclerotial bodies is attributed to Medlar (hence "Medlar bodies"), but since then the disease has been compared to other infections caused by dematiaceous fungi. With the introduction of the term "phaeohyphomycosis" (from ancient Greek Pheo = black) by Libero Ajello in 1974, the difference between these mycoses became more obvious. In 1992, the International Society for Human and Animal Mycology (ISHAM) recommended the adoption of the term "chromoblastomycosis", which Terra et al. coined in 1922, to define the pathology. The infection localizes in different parts of the body, following traumas resulting in penetra- 
tion or soiling with parasitized organic material. The most affected parts are feet and legs, secondarily hands, arms, face and chest. The species of dematiaceous fungi causing chromoblastomycosis are mainly $F$. pedrosoi and Cladophialophora carrionii, less frequently Phialophora verrucosa and Rhinocladiella aquaspersa, and even more rarely F. compacta, Exophiala spinifera, E. jeanselmei and E. (Wangiella) dermatitidis. F. pedrosoi and C. carrionii are common in the tropical and subtropical areas of the world. F. pedrosoi is also found in humid environments such as the Amazonian forests of Brazil and those of northern Madagascar, as well as in the temperate areas of Brazil, Uruguay and Argentina. $C$. carrioniiis is found in climatic zones characterized by modest rainfall in southern Madagascar, Australia, China, Caribbean (Mexico, North Venezuela, Cuba) and Africa. R. aquaspersa and P. verru$\cos a$ are responsible for chromoblastomycosis especially in South America. Cases of chromoblastomycosis have also been reported in the USA, Colombia, Dominican Republic, Japan and Malaysia. In Europe, quite exceptional cases have been reported in Italy, Spain, Russia, Finland, Romania, East Germany and in former Czechoslovakia. Young and adult male subjects working as farmers in rural areas are mainly affected.

\section{Zygomycosis due to Entomophthorales}

Fungi belonging to Entomophthorales are Zygomycetes that cause a chronic inflammatory disease affecting the subcutaneous tissues or the nasal mucosa. As they do not invade the vessels, they cause less damages compared to those caused by Mucorales. Entomophtoromycoses are mainly caused by fungi belonging to the genus Conidiobolus (C. coronatus and C. incongruus): they affect adult males, involving nose submucosa (rhinoentomophtoromycosis) and submucosal tissue of neighboring areas. Only rarely, they can determine systemic forms. They are observed in tropical areas (West Africa but also South America and India) where they affect not only in human but also animals (horses and sheep). Entophtoromycoses caused by the genus Basidiobolus (B. ranarum) are mainly detected in children and male adolescents affecting limbs, trunk, thorax, back and buttocks. These are subcutaneous nodules that subsequently give rise to extensive subcutaneous masses, hardened but painless. Fungi belonging to Basidiobolus spp are also reported affecting cold-blooded animals (reptiles and amphibians) especially in Africa.

\section{Lobomycosis}

The etiologic agent is Lacazia (Loboa) loboi: it is a non-cultivable fungus whose habitat is represented by the tropical areas of Central and South America. Lobomycosis is hyperendemic in some areas of Brazil, although some cases have recently been reported in Europe, South Africa, Canada and the USA. The first description was made in 1930 in Recife (Brazil) by J.O. Lobe (hence the name) that confused this pathology with a variant of blastomycosis ("Nova especies de blastomycose"). Predisposing condition is the typical environment of the luxuriant tropical areas, with average temperatures of $25^{\circ} \mathrm{C}$, rainy forest, high hydrometric indices $(<2000 \mathrm{~mm})$ and relative humidity $(>75 \%$ ), as well as low altitude (within $200-$ $250 \mathrm{~m}$ asl). Rare in humans, it is relatively frequent in dolphins such as Tursiops truncatus (bottle-nosed dolphin) and Sotalia guianensis (Guiana dolphin). Conidia of L. loboi observed in the histological preparations (fungus is not cultivable) are smaller in dolphins than in humans: for this reason, some authors suggest that the two microorganisms are different even if, conversely, serological data seem to confirm their identity. The possibility that contamination occurs through contact with dolphins seems low: there is only one case of lobomycosis detected in a dolphinarium worker (publication from the 1970s), probably contracted following a trauma: the lesions initially have a keloid aspect and, subsequently, take on a warty and then ulcerated course.

\section{E. Sporotrichosis}

It is a subacute or chronic mycotic ubiquitous infection of humans and some animals, caused by a dimorphic fungus: Sporothrix schenkii var. schenkii. Only more rarely S. schenkii var. luriei is involved. It affects skin, subcutaneous tissue and lymphatic vessels; sometimes it can involve bones and viscera. A disseminated form starting from the skin or lungs is rarely observed. The distinctive histopathological marker is represented by the "Splendor-Hoeppli phenomenon". S. schenkii is present in the soil and on decaying plants, thorny plants such as roses (the rosebushes disease), sphagna (mosses) and animals (armadillo). S. schenkii is more common in tropical and warm areas of Central and South America temperate areas. Additionally, there are hyperendemic areas with unusually high prevalence of infection (Brazil), but it is known as an autoctonous disease also in Europe, including Italy. The var. luriei has been isolated in rare cases, found in America, India and Italy. In the past, between 1930 and 1940, epidemic outbreaks were reported among the miners of the gold mines of South Africa. Outbreaks have also been reported among florists and, more rarely but in connection with the fungi particular habitat, among armadillos hunters or, more simply, in people who get into contact with domestic cats. Some patients remember to have experienced minor traumatism during work or gardening, although in the vast majority of cases the infection was not evident. Subjects suffering from sporotrichosis are usually in good health, while people with AIDS are more susceptible to becoming infected and can develop disseminated forms. The lymph-cutaneous form is the most frequent, usually following a traumatic event. It spreads through the lymphatic system, giving rise to a series of nodules that subsequently ulcerate, thus causing a purulent discharge. Hands, face, neck, trunk are the most affected areas; sometimes it complicates with the involvement of bones and viscera. The cutaneous form is usually primary: it remains localized at the implant site without lymphatic involvement (fixed cutaneous sporotrichosis). There are also muco-cutaneous, conjunctival and paranasal sinuses forms, the disseminated form in defeated subjects and, finally, the usually primitive pulmonary form contracted through conidia inhalation.

\section{F. Phaeohyphomycosis}

The term "Phaeohyphomycosis" was coined by Libero Ajello in 1974. It regards the painless subcutaneous nodule that evolves into a cyst, tending to ulcerate with the conclusive production of warty lesions, due to the penetration of black fungi into the subcutaneous tissues, following minor or inconsistent traumas. Subcutaneous localization represents the most frequent form of phaeohyphomycosis. Its etiological agents are mainly telluric saprophitic fungi, mostly ubiquitous, often with a low virulence: Curvularia (Bipolaris) spicifera, Alternaria alternata, A. longipeis, but also real pathogens such as E. jeanselmei and E. dermatitidis. The following are other etiological agents: Exserohilum rostratum, P. parasitica, P. richardsiae, Cladosporium spp, Veronaea botryosa. The most frequently affected subjects are adult males working in rural areas (farmers, gardeners). An important risk factor is also the immunosuppression related to solid organ transplants (a few dozen cases have been described). Immunodeficiency also justifies the evolution of initially subcutaneous forms to dissemination in other organs. A case of nosocomial infection due to E. rostratum has also been described, probably due to skin irritation through contamination of the venipuncture kit. 


\section{G. Hyalohyphomycosis}

Hyalohyphomycoses of the subcutaneous tissue are due to the coexistence of a double risk factor: immunosuppression (for solid organ transplantation: kidney or liver; for leukemia or lymphoma; for chronic granulomatous disease or diabetes) and rural activities. Skin manifestations occur with pictures of cellulite with ulceration, or erythematous papules and nodules, such as those supported by the different species of Paecilomyces and Trichoderma. The primitive cutaneous forms from hyaline fungi are caused by microorganisms belonging to the species: Fusarium solani, Aspergillus fumigatus, A. flavus, A. terreus, A. versicolor, Scopulariopsis brevicaulis, Purpureocillium liliacinus, Acremonium spp. The primitive forms are rare and observed almost exclusively in immunocompromised subjects.

\section{H. Zygomycosis due to Mucorales}

Zygomycetes are ubiquitous fungi whose conidia can be inhaled with lung and upper airways colonization. More exposed are those subjects affected by decompensated diabetes mellitus, neutropenic, immunocompromised, nephropathic, patients treated with deferoxamine. The fungus can invade the blood vessels with subsequent thrombosis and infarction of the surrounding tissues: these septic thrombi can embolize, spreading the infection in distant districts, causing craniofacial, rhinocerebral, lung, gastrointestinal forms, but also primitive and disseminated skin ones. In the 1970s, a study reported an epidemic of cutaneous mucormycosis caused by Rhizopus microsporus var. rhizopodiformis which had contaminated some lots of occlusive adhesive bandages used in post-surgical wounds. This evidence is the most obvious example of the possibility of mechanical contamination, which today is more frequently associated with major traumatisms, such as "road trauma". Other etiological agents of cutaneous mucormycosis are: Lichtheimia (Absidia) corymbifera, Apophysomices elegans, Mucor hiemalis, Rhizopus oryzae and Saksenaea vasiformis.

Some cases of subcutaneous zygomycosis have recently been described as a consequence of trauma resulting from natural disasters (Joplin tornado, TX) or war events (American military stationed in Iraq/Afghanistan).

\section{Subcutaneous dermatophytosis}

Dermatophytic infections are by definition skin mycoses, without involvement of the dermis deep layers and subcutaneous tissue. However, some studies have reported variants in which keratinophilic dermatophyte develops in depth, thus causing the appearance of granulomas. In particular, Majocchi's trichophyticum granuloma (described, in fact, by Domenico Majocchi in 1883 as "trichophyticum granuloma") is observed in defeated subjects or affected by immunological deficits. The most frequent etiological agents are: T. rubrum (above all), T. violaceum, T. mentagrophytes, M. canis, M. audouinii, M. gypseum and $M$. ferrugineum. In the initial phase, the fungus parasites hair, then deepens into the pilo-sebaceous unit and, through the rupture of the follicular wall, disseminates into the dermis. In this first phase, erythemato-edematous lesions are clinically observed which then cause papular lesions tending to increase in number and size. In the full-blown phase, nodular lesions are observed, sometimes even very large, single or united in plaques, covered with erythematous alopecic skin.

Other forms of granuloma are also described:

- Dermatophytic pseudomycetoma: clinically characterized by more or less raised lesions on the skin with the formation of fistulas secreting white grains of $100 \mu$ in diameter. Generally, these patients present with a superficial dermatophytosis from which the Dermatophytes invade the subcutaneous tissue. Therefore, in this particular form of mycetoma, there is no traumatic inoculation (dermatophytic pseudomycetoma). The etiological agents are: T. rubrum, T. mentagrophytes, M. canis, M. audouinii, M. gypseum, and M. Langeronii.

- Wilson's dermatophytosis (legs granulomatous tinea): It generally affects middle-aged women with circulatory system problems and it is often caused by depilatory practices that determine the penetration of the infected hair into the dermis, with the formation of either single or gathered in plaques lesions. Granulomas difficult to be identified require a differential diagnosis with nodular erythema, erythema induratum of Bazin and nodular vasculitis. The etiological agent is typically T. rubrum. The pathology affects immunodeficient subjects (T lymphocyte deficiency) or debilitated for serious deficiency states or severe hormonal dysfunctions. There is a particular genetic predisposition to develop dermatophytic disease: subjects (few cases) of North African ethnicity (in particular Algeria) are affected. They could manifest extensive varied dermatophytosis with fungi invading the subcutaneous tissue through the lymphatic pathways. This involves the formation of granulomas tending to flow into large plates with lymphoedema, softening, sometimes necrosis, draining ulcerations. It might happen that the lesions, after reaching the draining lymph nodes, also spread to other skin areas or to deep organs (including brain and liver) with fatal outcome.

\section{J. Secondary subcutaneous manifestations of systemic mycoses}

Many species, responsible for disseminated or deep forms, can reach the skin and subcutaneous tissues (yeast, hyalohyphomycetes, phaeohyphomycetes, zygomycetes and dimorphic fungi) by lymphatic pathway, defining secondary localization.

In particular:

- Yeasts: The metastatic skin lesions occur in about $10 \%$ of patients with disseminated infection by Candida spp or other yeasts or in the course of yeast fungemia. They affect immunocompromised, neoplastic, debilitated, diabetic subjects. $C$. albicans is responsible for at least $50 \%$ of these metastatic forms affecting the skin; the other yeasts involved belong to the genera Candida, Trichosporon, Cryptococcus (C. neoformans) and, to a lesser extent, other genera.

- Hyalohyphomycetes: Several are the genera and species that can lead to deep mycosis in humans; however, fungi that very often present a secondary skin involvement belong to the genera Aspergillus (A. fumigatus, A. flavus) and Fusarium (F. solani). Immunocompromised and especially neutropenic patients are affected.

- Phaeohyphomycetes: These forms are supported by dematiaceous fungi (E. rostratum, Curvularia (Bipolaris) spicifera, C. lunata, C. pallescens, E. jeanselmei, A. alternata, A. longipeis, $V$. botryosa). Again, immunocompromised individuals are affected.

- Zygomycetes: Dissemination of mucormycosis is not frequent but always lethal. R. pusillus, R. oryzae, S. vasiformis are the species involved. They affect diabetic, nephropathic, neutropenic, debilitated, neoplastic and AIDS subjects.

- Dimorphic fungi: a) Histoplasma capsulatum: It causes chronic granulomatous disease starting with an early pulmonary localization $H$. capsulatum var. capsulatum is frequently isolated from the faeces of birds and bats, which represent the primary source of infection. In Africa, cases due to H. capsulatum var.duboisii have been reported. b) Coccidiodes posadasii/immitis: With the exception of California (where the 
pathology is caused by $C$. immitis), elsewhere the causative agent is $C$. posadasii, from the name of the Argentine physician (A. Posadas) who first diagnosed a case of coccidioidomycosis. It is found in the desert areas of the United States, Mexico, Central America, South America. c) Blastomyces dermatitidis: It lives near the waterways of some areas of Canada, the United States, Israel, Saudi Arabia, Europe (Poland), Africa, India. d) Paraccocidiodes brasiliensis: Some case have been reported in Mexico and Central and South America countries. Fungus distribution seems to be correlated to that of the nine-banded armadillo (Dasypus novemcinctus) which is also affected by the infection. e) Talaromyces (Penicillium) marneffei: It is endemic in Southeast Asia. Probable reserve of infection is the bamboo rat. Unlike other dimorphic fungi (all primary pathogens), it is an opportunistic pathogen and affects immunocompromised patients, in particular those affected by AIDS.

\section{Diagnosis methods}

Since the fungus etiological diagnosis can require prolonged times, it is essential that the laboratory reports step by step, to the clinician all the preliminary information which could beuseful for the proper therapeutic approach.

\section{Mycetoma}

It is essential to alert microbiologists of the suspected diagnosis, so that they can adopt the specific diagnostic procedures for the fungi detection from culture, that could require very long incubation periods (not less than one month). The diagnosis is often difficult at least at the first clinical signs, as it could be wrongly confused with other infectious pathologies (osteomyelitis, tuberculosis, phaeohyphyomycosis or chromoblastomycosis) and, above all, with pictures of bone or subcutaneous neoplasia (melanoma or Kaposi's sarcoma). The diagnostic process requires that:

- The specimen (pus or biopsy material) should be quickly examined for the "grains" detection. The histological examina- tion of the grain ("black" or "white") is the most reliable criterion for the etiological diagnosis. The tissue reaction and the grain morphology, observed after hematoxylin-eosin staining (possibly together with silver stain methods according to Gomori-Grocott or PAS) allow for an etiological diagnosis, as briefly reported in Tables 1 and 2 .

- The mycological culture can be carried out from the grains washed and stored for 18 hours in antibiotic solution, or from biopsy material stored in a physiological solution with $20 \%$ glycerin: 3-4 grains should be inoculated in a tube containing Sabouraud agar enriched with antibiotics (chloramphenicol) but without actidione, arranging them about $2 \mathrm{~cm}$ away from each other. Two sets of a dozen tubes should be inoculated; with an incubation period of about 2 months at $30^{\circ} \mathrm{C}$ and $37^{\circ}$ $\mathrm{C}$, respectively. Additionally, in case of dematiaceous fungi the colonies color is gray-black, olive green or intensely brown on recto and black or intensely dark on verso. It should be noted that, besides Acremonium spp. and N. rosatii, Aspergillus spp., Fusarium spp. and, exceptionally, dermatophytes can also be isolated in culture. The microscopic examination of the colonies highlights different aspects that allow their identification of genus and species.

- Serological investigations: not available.

- Immunological methods: not available.

- Biomolecular techniques: not available.

\section{Chromoblastomycosis}

Microbiologists should be alerted about the suspected diagnosis, so that they can adopt the specific diagnostic procedures for black fungi detection, whose cultivation requires very long incubation periods (not less than one month). Clinical diagnosis is often difficult; a differential diagnosis should be made with other infectious diseases (anthrax, leishmaniasis, cutaneous tuberculosis) and, above all, cutaneous neoplastic pictures. The diagnostic process requires that:

1. The sample (pus or biopsy material) should be immediately examined. The histological examination (PAS or GrocottGomori staining) is suggestive of chromoblastomycosis if the 'sclerotic bodies' are observed. In particular, the histological exam and the direct observation of the epidermal lesion, after

Table 1. Features of black grains.

\begin{tabular}{lllll} 
Texture & Form & Dimensions & Histology (E-E) & Etiology \\
Soft & Irregular, thick & $>0.5 \mathrm{~mm}$ & Empty spheroid, or vermiform sinuous bands & E. jeanselmei \\
Hard & Spheroidal & $1-2 \mathrm{~mm}$ & Empty spheroid & L. senegalensis \\
\hline $\begin{array}{l}\text { Soft } \\
\text { Hard }\end{array}$ & Spheroid curved & $>0.5 \mathrm{~mm}$ & Peripheral ring of hyphae and pigment with central pale area & M. grisea \\
& $\begin{array}{l}\text { Spheroidal, oval, with } \\
\text { moriform aggregations }\end{array}$ & $>0.5 \mathrm{~mm}$ & Compact, with hyphae and 'vesicular' cells & M. mycetomatis \\
\hline Soft & Spherical or tubular & $0.3-1 \mathrm{~mm}$ & $\begin{array}{l}\text { Curved ring with hyphae in the periphery and pigment, } \\
\text { and with a central pale area }\end{array}$ & P. romeroi \\
\hline
\end{tabular}

Table 2. Features of white grains.

\begin{tabular}{llll} 
Texture & Form & Dimensions & Histology (E-E) \\
Soft & Round or oval & $0.5-2 \mathrm{~mm}$ & Regular \\
Soft & Globose & $0.5-1 \mathrm{~mm}$ & Compact, with peripheral hyphae and central "vesicular" cells \\
\hline
\end{tabular}


the specimen clarification with $30 \%$ potassium hydroxide reveal a hyperplasia with papillomatosis, hyperkeratosis and hyperacantosis. At the level of Malpighi's mucous bodies, horny globes and micro-abscesses rich in neutrophil are noted. Conversely, the direct examination of the dermal lesion shows a granulomatous reaction with giant Langhans cells and highly polymorphic cell infiltrates, with histiocytes, neutrophils and plasma cells. At the dermis base, on the contrary, a stroma with variable features (edematous, fibrous, mixed) is detected. The 'sclerotic bodies' (or 'fumagoid cells)' are pathognomonic. These are brown, septate and muriform structures, with a diameter between 7 and $15 \mu$, corresponding to the fungal element, mainly present in the dermis but also in the epidermis, where they emerge through a trans-epithelialization mechanism, free or inside the horny globes or giant cell.

2. The mycological culture can be carried out from the crusts, scales or biopsy material. The culture on Sabouraud agar enriched with antibiotics (chloramphenicol) requires very long incubation periods (never less than one month) at temperatures between $20^{\circ}$ and $30^{\circ} \mathrm{C}$. The fungal colonies are finely velvety and over time acquire a hard consistency. The colonies color is gray-black, olive green or intensely brown on the recto and black or intensely dark on the verso. The microscopic examination of the colonies highlights different aspects that allow for the identification at the genus and species level. Finally, it is possible to induce the fungus switchting from the filamentous to the yeast-shaped (fumagoid) form by using Francis medium enriched with horse blood or Sabouraud liquid medium at $\mathrm{pH}$ 2.5 and at $37^{\circ} \mathrm{C}$. Such procedure, however, is only performed by Reference Centers.

3. Serological investigations: not available for routine diagnostics.

4. Immunological methods: not usually available. They can only be helpful in endemic areas. However, the intradermal reaction with $F$. pedrosoi exoantigen, as well as immunofluorescence, immunoprecipitation and immunoenzymatic techniques are not routinely available.

5. Biomolecular techniques: not available.

\section{Zygomycosis due to Entomophthorales}

Microbiologists should be alerted on the diagnostic suspect, in order to adopt the ad hoc diagnostic procedures aimed at recovering Entomophtorales. In our climate, this pathology is almost absent. In case of suspected diagnosis, it is advisable to refer to a Reference Center. The diagnostic process requires that:

1. The biopsy material should be examined as soon as it arrives to the Laboratory. The histological examination (PAS or Grocott-Gomori staining) is suggestive of entomophthorosis if cenocytic (non septate) hyphae are noted.

2. The mycological culture requires inoculation of numerous tubes containing Sabouraud agar enriched with antibiotics (chloramphenicol) but without actidione incubated at temperatures between $30^{\circ}$ and $37^{\circ} \mathrm{C}$. Fungal colonies are characterized by the presence of ejectable spores that are detected on the plate cover or the opposite side the medium slant. Species identification is achieved by the means of the enzymatic activity evaluation of the isolated colonies (trypsin and beta-glucuronidase).

3. Serological investigations: not available for routine diagnostics.

4. Immunological methods: not available for routine diagnostics.

5. Biomolecular techniques: not available for routine diagnosis.

\section{Lobomycosis}

Microbiologists (and especially the anatomopathologist) should be alerted about the diagnostic suspect on the basis of the clinical picture. The causative agent Lacazia (Loboa) loboi is a non-cultivable fungus. The diagnostic process requires that:

1. The histological examination (PAS or Grocott-Gomori staining) is suggestive of lobomycosis if thick-walled yeast cells, arranged in chains connected by an isthmus, are found.

2. The culture constantly gives negative results.

3. Serological investigations: not available for routine diagnostics.

4. Immunological methods: not available for routine diagnostics.

5. Biomolecular techniques: not available for routine diagnostics.

\section{Sporotrichosis}

Microbiologists should be alerted about the diagnostic suspect based on the clinical presentation, so that they can adopt the ad hoc diagnosis procedures for $S$. schenckii culture detection. Actually, the clinical diagnosis is often difficult, initially appearing as a normal bacterial infection. The ulcerated forms of "sporotrichotic cancer" must be compared with other infectionrelated diseases (anthrax, leishmaniasis, cutaneous tuberculosis, Buruli ulcer, cutaneous amoebiasis and larva migrans cutanea). Warty lesions must be differentiated from skin neoplasms, chromoblastomycosis, erythematous scaly type and dermatophytic pathology.

The diagnostic process requires that:

1. Samples (pus or biopsy material) should be immediately examined, even though most of times this observation is negative. The histological examination (PAS or Grocott-Gomori staining) is suggestive of sporotrichosis if the eosinophilic "asteroid bodies" are highlighted (Hoeppli-Splendor phenomenon).

2. The culture examination is positive, if incubated at two temperatures (environmental and $37^{\circ} \mathrm{C}$ ) for long time.

3. Serological investigations: useful (microagglutination test), but not easily available.

4. Immunological methods: sporotrichin intradermoreation is seldom used.

5. Biomolecular techniques: not available.

\section{Phaeohyphomycosis}

Since black fungi have a ubiquitous distribution and are frequently found as contaminants, even in the laboratory environment, the diagnosis of phaeohyphomycosis can only be based on the classical criteria of mycological diagnostics. The histological evidence (Fontana-Masson melanin-specific staining must be preferred because, especially at the onset, hyphae may not be pigmented, thus resulting in falsely negative results) of yeast-like forms, as well as hyphae and positive culture. The diagnostic process requires that:

1. Samples (pus or biopsy material) should be immediately examined Samples withdrawn by biopsy, then homogenized in sterile saline solution $0.9 \%$, are preferred. When such molds (such as: Alternaria spp., Curvularia spp, Lasiodiploidea theobromae, Onychocola canadensis, Phialophora spp, Phyllosticta spp,) are identified in culture, it is recommended to repeat sampling in order to confirm the 
presence of black fungi in serial specimens and, therefore, consider their role appropriately. Diagnosis of the dermal lesions first of all requires the histological examination. It highlights the presence of fungi inside an inflammatory granuloma located in the middle or upper layers of the dermis, which can evolve in an abscess. Such fungi may appear both in a filamentous form (hyphae, 5-10 $\mu$ in diameter) and yeastshaped, with ovoid or globose features. Finding of the foreign body (e.g., thorns) is not accidental. The phaeohyhomycotic cysts require a histological exam: the preparation highlights, inside an intense fibrotic reaction, an abscess heavily constituted by neutrophils, eosinophils and lymphocytes, giant cells, sometimes vegetal residue and fungus in a filamentous, vesicular form, yellow-brown in color.

2. Samples withdrawn by biopsy, then homogenized in sterile $0.9 \%$ solution. For etiological diagnosis, it is necessary to perform the culture that allows for the isolation of dematiaceous fungi, from the cysts liquid or from the biopsy fragment. Although Exophiala (in particular E. jeanselmei, but also E. moniliae, E. pisciphila and E. spinifera) and Phialophora genera (P. bubackii, P. europeae, $P$. parasitica, $P$. repens, $P$. richardsiae, $P$. verrucosa) are prevalent, anecdotal evidence of the following fungi is more and more frequent: Cladosporium devriesii, Ochroconis galloparvum, P. dematioides, Phoma minutella, Pleurophomopsis lignicola, Taeniolella exilis, Tetraploa aristata, V. botryosa.

3. Serological investigations: useful (microagglutination test), but not easily available

4. Immunological methods: intradermoreaction to sporotrichin is rarely used

5. Biomolecular techniques: not available.

\section{Hyalohyphomycosis, Zygomycosis and Dermatophytosis}

Considering the ubiquitous distribution of hyaline filamentous fungi and their frequent recovery as contaminants, even in the laboratory environment, the diagnosis of hyalohyphomycosis must be based on the 'classic' criteria of mycological diagnostics: histological evidence and culture confirmation. The diagnostic work flow requires that:

1. Samples (pus or biopsy material) should be immediately examined. The quality of the biological material withdrawn (samples taken by biopsy, then homogenized in $0.9 \%$ sterile saline solution are preferred) as well as the assessment rapidity are mandatory.

2. Hyaline filamentous fungi are generally characterized by a rapid growth in vitro (4-6 days). The colonies macroscopic features are very heterogeneous with species-specific variation of texture and color, also significantly affected by experimental conditions (culture medium, incubation temperature). Identification to the genus and species level is based on microscopic morphology (conidiophores, conidiogenous cells and conidia). Cultivations should be performed using simple media incubated for 48 hours at $35^{\circ} \mathrm{C}$, followed by a further incubation for 5 days at $30^{\circ} \mathrm{C}$. For identification, resistance or susceptibility to cycloheximide, and any metabolic characteristic of thermotolerance may be relevant.

3. Serological investigations: useful (microagglutination test), but not easily available

4. Immunological methods: intradermoreaction is rarely used

5. Biomolecular techniques: not available.

\section{References}

Ajello L, Farina C, Mazzoni A, Picerno G. Eds.: "Fondamenti di Micologia Clinica” AMCLI Ed.

Andreoni S, Farina C, Lombardi G. Atlas of Medical Mycology. System Ed 2005.

Badillet G. Dermatophytes et immigration. Ann Biol Clin 1988;46:37-43.

Clancy CJ, Wingard JR, Nguyen MH. Subcutaneous phaeohyphomycosis in transplant recipients, review of the literature and demonstration of in vitro synergy between antifungal agents. Med Mycol 2000;38:169-75.

Denis J, Machouart M, Morio F, et al. Performance of matrixassisted laser desorption ionization-time of flight mass spectrometry for identifying clinical Malassezia isolates. J Clin Microbiol 2017;55:90-6.

Di Silverio A, Borelli D. Malattie cutanee da miceti. In F. Serri (ed.), "Trattato di Dermatologia". Vol. II, Editore Piccin Padova, 1987.

Drouhet E, Boiron P. Metodi e tecniche per la diagnosi di laboratorio in micologia medica. In: Trattato Italiano di Medicina di Laboratorio fondato da A. Burlina, Vol III/2: Microbiologia Clinica, A. Eyquem, J. Alouf, L. Montagnier (eds.) (Traduzione Italiana di O. Zangaglia); Cap 115, pag 10791109, Piccin Nuova Libraria S.p.A., Padova 1999.

Elewski BE, Hazen PG. The superficial mycoses and the dermatophytes. J Am Acad Dermatol 1989;21:65:673.

El Hag A, Fahal AH, Khalil EA. Fine needle aspiration cytology of mycetoma. Acta Cytol 1996;40:461-4.

Georg LK, Camp LB. Routine nutritional tests for the identification of dermatophytes. J Bacteriol 1957;74:113-21.

Hay RJ. Dermatofitosi e altre micosi superficiali. In: GL Mandell, RG Douglas Jr., JE Bennett (eds.), "Malattie infettive. Principi e pratica" Vol.II cap.245, pag. 2546-2558, Piccin Nuova Libraria, Padova, 1993

Hay RJ, Roberts SOB, Mckenzie DWR. In: Rook/Wilkinson/Ebling's Textbook of Dermatology. RH Champion, JL Burton, FJG Ebling eds. Blackwell Scientific Publications, Oxford, 1998 - 6th edition, Cap 27, pag. 11631170. (ed.), "Immunology of fungal diseases". Marcel Dekker, Inc., New York.

Hayette MP, Seidel L, Adjetey C et al. Clinical evaluation of the DermaGenius $^{\circledR}$ nail real-time PCR assay for the detection of dermatophytes and Candida albicans in nails. Medical Mycology 2019; 57: 277-283.

Kane J, Summerbell RC, Sigler L, Krajden S, Land GA. Laboratory handbook of dermatophytes. Star Publishing, Belmont, Calif 1997.

Koneman EW, Roberts GD. Practical Laboratory Mycology. $3^{\text {rd }}$ Edition The Williams \& Wilkins Company, Baltimore, 1985.

Kwon-Chung KJ, Bennett JE. Medical mycology. Lea \& Febiger, Philadelphia 1992.

Larone DH. Medically important fungi, A guide to identification. $5^{\text {th }}$ Edition. ASM Press, Washington 2011.

Matsumoto T, Ajello L, Matsuda T, et al. Developments in hyalohyphomycosis and phaeohyphomycosis. J Med Vet Mycol 1994;32:329-49.

Mcginnis MR. Chromoblastomycosis and phaeohyphomycosis: new concepts, diagnosis, and mycology. J Am Acad Dermatol 1983;8:1-16.

Panconesi E, Difonzo EM. Tranelli di una facile diagnosi: le micosi superficiali. Micol Dermatol 1993;7:3-13

Pinetti P. Le dermatofizie. Piccin Editore, Padova, 1977. 


\section{Study Protocol}

Polonelli L, Ajello L, Morace G. Micologia Medica. Società Editrice Esculapio 1993.

Rebell G, Taplin D. Dermatophytes their recognition and identification. University of Miami Press, Coral Gables, Fla, 1970.

Rippon JW. Medical Mycology. The pathogenic fungi and the pathogenic actinomycetes, 3rd ed. WB Saunders, Co, Philadelphia, 1988.

Salkin IF, Padhye AA, Kemna ME. A new medium for the presumptive identification of dermatophytes. J Clin Microbiol 1997;35;2660-2.

Taplin D, Zaias N, Rebell G, Blank H. Isolation and recognition of dermatophytes on a new medium (DTM). Arch Derm 1969;99:203-9.

Tosti A, Baran R, Piraccini BM, Fanti PA. Endonix onychomycosis: a new modality of nail invasion by dermatophytes. Acta Derm Venereol 1999;79:52-3.

Tosti A, Piraccini BM. Le onicomicosi. Atlante a colori. Medhoc Editore Milano, 1996. pag 89-106.

Uribe F. Histopathology of chromoblastomycosis. Mycopathologia 1989;105:1-6.

Weitzman I, Summerbell RC. The dermatophytes. Clin Microbiol Rev 1995;8:240-59. 